\title{
R-Process nucleosynthesis in high entropy environment in explosion of supernova type II and neutron star formation
}

\author{
Rulee Baruah ${ }^{1}$, Kalpana Duorah ${ }^{2}$ and H. L. Duorah ${ }^{2}$ \\ ${ }^{1}$ Inst. of Engineering and Technology, Jorhat-785001, India \\ email: ruleeb@yahoo.com \\ ${ }^{2}$ Dept. of Physics, Gauhati University, Guwahati-781014, India.
}

\begin{abstract}
It is generally acknowledged that Type II supernovae result from the collapse of iron core of a massive star which, at least in some cases, produces a neutron star. At this stage, the neutrinos are produced by neutronization which speeds up as collapse continues. During collapse an outward bound shock wave forms in the matter falling onto the nearly stationary core. The conditions behind the shock at 100 to $200 \mathrm{~km}$ are suitable for neutrino heating. This neutrino heating blows a hot bubble above the protoneutron star and is the most important source of energy for Supernova explosion. At this stage, we try to attain the r-process (rapid neutron capture process) path responsible for the production of heavy elements beyond iron, which are otherwise not possible to be formed by fusion reactions. The most interesting evolution occurs as temperature falls from $10^{10} \mathrm{~K}$ to $10^{9} \mathrm{~K}$. At these high temperature conditions, the critical fluids after fusion reactions are forbidden and transform into the respective atoms by r-process path which on beta decaying produce the ultimate elements of the periodic chart.

Another astrophysical parameter needed for our analysis is neutron number density which we take to be greater than $10^{20} \mathrm{~cm}^{-3}$. With these, at different entropy environments, we assign the neutron binding energy that represents the r-process path in the chart of nuclides. Along the path, the experimental data of observed elements matches our calculated one. We find that an entropy of $\sim 300$ with $\mathrm{Ye} \simeq 0.45$ can lead to a successful r-process. It produced heavy neutronrich nuclei with $\mathrm{A} \simeq 80-240$. Later ejecta are neutron-rich $(Y e \leqslant 0.5)$ and leaves behind a compact neutron star.
\end{abstract}

